\title{
Commentaire
}

\section{Améliorer notre compréhension de l'épidémie de surdoses liées aux opioïdes de médicaments et de drogues illicites contaminées au Canada}

\author{
Robert Strang, M.D., M. Sc. S., FRCPC
}

Diffuser cet article sur Twitter

J'ai le privilège de présenter ce second numéro spécial de Promotion de la santé et prévention des maladies chroniques au Canada : Recherche, politiques et pratiques consacré à la crise des opioïdes qui sévit actuellement au Canada. En tant que médecin hygiéniste en chef de la NouvelleÉcosse, je m'occupe d'un large éventail de problèmes de santé publique, et la crise des opioïdes est l'un des problèmes les plus difficiles à cause de son ampleur, de sa portée et de sa nature complexe. Le bilan des décès est affligeant : en 2017, presque 4000 personnes au Canada ont perdu la vie en raison de surdoses apparemment liées aux opioïdes, et cette estimation pourrait augmenter avec la disponibilité progressive des données ${ }^{1}$. Toutes les régions du pays et tous les secteurs de la société canadienne sont touchés. La majorité de ces décès se produisent chez les hommes $(78 \%)$ et parmi les 30 à 39 ans $(28 \%)^{1}$. Les données indiquent que les surdoses apparemment liées aux opioïdes pourraient constituer la cause principale de décès chez les hommes de 30 à 39 ans $^{2}$. L'une des raisons que cette crise des opioïdes est si complexe est qu'elle est en fait constituée de deux épidémies liées, soit une épidémie de problèmes de santé dus aux opioïdes pharmaceutiques et incluant des surdoses, et une épidémie de surdoses de drogues illicites contaminées.

Quand les gouvernements provinciaux, territoriaux et fédéral ont créé le Comité consultatif spécial sur l'épidémie de surdoses d'opioïdes (CCS), que je copréside avec mon homologue fédérale, $\mathrm{D}^{\text {re }}$ Theresa Tam, administratrice en chef de la santé publique du Canada, nous ne savions même pas combien de Canadiens mouraient chaque année de surdoses d'opioïdes. La mise sur pied d'un système national de surveillance des décès apparemment dus aux opioïdes a été en soi une réalisation importante, qui a exigé une étroite coopération entre les secteurs de la justice, de la sécurité publique et communautaire et de la santé dans les 13 provinces et territoires et le gouvernement fédéral. Cette nouvelle collaboration nationale entre secteurs était nécessaire pour obtenir un résultat crucial, soit quantifier les décès causés par une surdose d'opioïdes, mais il ne constitue que l'un des nombreux partenariats nécessaires pour régler efficacement cette crise. Agir de manière efficace et coordonnée nécessite en effet la collecte d'un large éventail de données sans cesse actualisées, ce qui exige une collaboration entre le secteur de la santé publique et les secteurs gouvernementaux de la justice, de la sécurité publique et communautaire et de la sécurité frontalière (ce qui est inhabituel) ainsi qu'avec, entre autres, les premiers intervenants, les fournisseurs de soins de base, les agences de réduction des méfaits et les personnes ayant des expériences personnelles pertinentes. Nous disposons maintenant de données sur les déterminants immédiats de cette crise et la comprenons mieux. Par exemple, nous savons qu'en 2017, le fentanyl et ses analogues étaient en cause dans trois quarts (72 \%) des décès apparemment dus aux opioïdes et que, dans la majorité (71 \%) des décès accidentels, une ou plusieurs substances non opioïdes étaient en cause ${ }^{1}$. Cependant, il reste beaucoup à faire pour décrire et quantifier le réseau complexe de causes en amont de ces épidémies de surdoses d'opioïdes pharmaceutiques et de drogues illicites contaminées.

Les articles de ce numéro doivent ainsi être situés dans un contexte plus large : nous en savons plus qu'en décembre 2016, au moment où le CCS a été créé, mais nous avons besoin d'en savoir beaucoup plus pour réduire de manière importante le nombre de Canadiens qui décèdent de surdoses liées aux opioïdes de médicaments ou de drogues illicites. Collectivement, ces articles améliorent notre compréhension de la crise des opioïdes au Canada et aident à guider nos interventions en santé publique.

Les trois premiers articles examinent plusieurs initiatives de surveillance qui éclairent différents aspects de la crise dans une perspective nationale. Abdesselam et ses collègues $^{3}$ attirent l'attention sur des initiatives lancées par le gouvernement du Canada et des partenaires nationaux, provinciaux et territoriaux pour surveiller la crise des opioïdes, et ils préconisent des approches de surveillance novatrices pour améliorer la cueillette, l'analyse et l'harmonisation de données sur les médicaments et les drogues, comme la mise en place d'un observatoire national des drogues. Do et ses collègues ${ }^{4}$ utilisent les données nationales sur les visites aux services d'urgence liées à une intoxication aux opioïdes du Système canadien hospitalier d'information et de recherche en prévention des traumatismes (eSCHIRPT), un réseau de 6 hôpitaux généraux et de 11 hôpitaux pédiatriques à l'échelle du pays, pour examiner le contexte et les circonstances associés à ces visites. Leur étude fournit de premiers résultats sur l'influence des facteurs proximaux et distaux en amont liés à la consommation d'opioïdes et elle souligne que la santé mentale semble un facteur contributif. Do ${ }^{5}$ a également fait équipe avec les services paramédicaux de la Ville d'Ottawa pour valider l'idée d'une surveillance nationale des données des services médicaux d’urgence 
(SMU) sur les surdoses suspectées avant l'admission des victimes dans un établissement de santé. Leur validation démontre que les données paramédicales disponibles peuvent fournir une information riche dans les meilleurs délais.

Les trois articles suivants apportent des idées sur les possibilités d'intervention, que ce soit sur les facteurs en amont comme l'approvisionnement ou la santé mentale ou que ce soit en aval comme la mobilisation des systèmes de santé pour surveiller les méfaits dus aux opioïdes et leurs impacts sur la santé. En ColombieBritannique, Otterstatter et ses collègues ${ }^{6}$ décrivent, au moyen de la Cohorte provinciale des victimes de surdose de ColombieBritannique, les tendances dans l'utilisation des soins de santé chez les personnes ayant fait une surdose de drogues illicites ${ }^{6}$. Ils constatent que les cas de surdose sont associés à un taux plus élevé d'utilisation des services de santé, ce qui suggère qu'il y a dans le système de santé des occasions, manquées et à saisir, d'intervention et de prise de mesures préventives. En NouvelleÉcosse, Schleihauf ${ }^{7}$ et son équipe décrivent l'effet des changements dans les pratiques de prescription des opioïdes sur les taux de décès liés aux opioïdes. Ils ont ainsi découvert que, même si les taux de mortalité liée aux opioïdes sont restés plus ou moins les mêmes entre 2011 et 2017, le nombre d'unités de morphine d'ordonnance distribuées par les pharmaciens a diminué et que la proportion de décès attribués aux opioïdes non pharmaceutiques a augmenté à la suite de récents changements dans les lignes directrices sur les prescriptions. Cette découverte fait ressortir l'importance de surveiller à la fois les prescriptions et l'offre de drogues illicites afin de déterminer l'effet des changements des pratiques de prescription sur les tendances dans la consommation de drogues. En Alberta, $\mathrm{Chan}^{8}$ et ses collègues ont étudié les tendances des suicides avec opioïdes entre 2000 et 2016 ainsi que leur poids par rapport aux autres méthodes de suicide. Ils n’ont pas observé de tendances similaires entre les surdoses intentionnelles avec opioïdes (suicides) et les surdoses accidentelles avec fentanyl illégal, ce qui suggère que les victimes de suicide avec opioïdes diffèrent de celles qui décèdent de surdose accidentelle, et par conséquent que les interventions en santé publique doivent être adaptées à chaque catégorie de population.
Depuis deux ans, les gouvernements aux niveaux fédéral, provincial et territorial ont mobilisé des ressources financières et techniques pour répondre à la crise. Conformément aux quatre piliers de l'approche fédérale de la crise des opioïdes (prévention, traitement, réduction des méfaits, application de la loi) ${ }^{9}$, plusieurs provinces et territoires ont mis en place des plans et des stratégies d'action coordonnée. Si la surveillance en santé publique a été - et va continuer à être - essentielle pour apporter des réponses efficaces et coordonnées, elle ne devra cependant pas surveiller uniquement les résultats en matière de santé, la fourniture et la consommation de médicaments et de drogues illicites, mais aussi explorer les déterminants sociaux et structuraux plus profonds de la santé, c'està-dire "les causes des causes $»^{10}$. Pour comprendre les facteurs déterminants en amont, il va falloir qu'un plus grand nombre de secteurs se mobilisent pour s'investir activement dans l'élaboration et le ciblage de stratégies de prévention et d'intervention.

Le travail réalisé dans le domaine de la surveillance continue à fournir les données nécessaires pour éclairer la crise, et l'effort pour remonter à ses causes en amont est sans précédent. Les leçons tirées vont nous servir pour l'étude plus générale de la consommation de drogues, en particulier les effets de la future législation sur le cannabis.

J'aimerais profiter de l'occasion pour féliciter toutes les personnes qui travaillent en santé publique et dans les disciplines connexes pour avoir collaboré avec détermination afin de colliger et analyser les données, inspirer et soutenir le travail en réponse à ces épidémies.

\section{$D^{r}$ Robert Strang \\ Médecin hygiéniste en chef \\ Ministère de la Santé et du Mieux-être de la Nouvelle-Écosse}

\section{Références}

1. Comité consultatif spécial sur l'épidémie de surdoses d'opioïdes. Rapport national : Décès apparemment liés à la consommation d'opioïdes au Canada (de janvier 2016 à décembre 2017). Ottawa (Ont.) : Agence de la santé publique du Canada; 2018 (consulté en juillet 2018). En ligne : https://www .canada.ca/fr/sante-publique/services /publications/vie-saine/rapport-national -deces-apparemment-lies-consommation -opioides-publie-juin-2018.html
2. Annonce de l'administrateur en chef de la santé publique du Canada à la séance d'information technique conjointe de l'Agence de la santé publique du Canada et des Instituts de recherche en santé du Canada, 19 juin 2018. Ottawa : (Ont.) : ASPC; 2018.

3. Abdesselam K, Dann M J, Alwis R, et al. Aperçu - Crise des opioïdes : assurer le suivi et faire évoluer les données de surveillance. Promotion de la santé et prévention des maladies chroniques au Canada. 2018;38(9):353-358.

4. Do M, Chang V, Tibebu S, et al. Surveillance des cas suspectés d'intoxication et de blessures liées aux opioïdes : tendances et contexte tirés du Système canadien hospitalier d'information et de recherche en prévention des traumatismes, mars 2011 à juin 2017. Promotion de la santé et prévention des maladies chroniques au Canada. 2018;38(9): 359-370.

5. Do M, Furlong G, Leyenaar M, et al. Aperçu - Que peuvent nous révéler les données des ambulanciers paramédicaux sur la crise des opioïdes au Canada? Promotion de la santé et prévention des maladies chroniques au Canada. 2018;38(9):384-387.

6. Otterstatter M, Crabtree A, Dobrer S, et al. Habitudes d'utilisation des soins de santé chez les personnes ayant fait une surdose de drogues illicites : analyse descriptive fondée sur la Cohorte provinciale des victimes de surdoses de la Colombie Britannique. Promotion de la santé et prévention des maladies chroniques au Canada. 2018;38(9):371-377.

7. Schleihauf E, Crabtree K, Dohoo C, et al. Aperçu - Surveillance simultanée des pratiques de prescription d'opioïdes et des décès liés à l'usage d'opioïdes : le contexte en NouvelleÉcosse (Canada). Promotion de la santé et prévention des maladies chroniques au Canada. 2018;38(9):378-383.

8. Chan E, McDonald B, Brooks-Lim E, et al. Aperçu - Suicides et intoxication aux opioïdes en Alberta (20002016). Promotion de la santé et prévention des maladies chroniques au Canada. 2018;38(9):388-392. 
9. Gouvernement du Canada. Mesures du gouvernement du Canada sur les opioïdes : 2016 et 2017, Ottawa, 2017 (consulté en juillet 2018). Sur Internet : https://www.canada.ca/fr/sante -canada/services/publications/vie -saine/mesures-opioides-2016-2017 .html

10. Marmot M. Social determinants of health inequalities. Lancet. 2005; 365(9464):1099-1104. 